\title{
OPTIMISATION ET PLANIFICATION
}

\section{DES RESEAUX DE TELECOMMUNICATIONS}

M. MINOUX

Centre National d'Etudes

des Télécommunications

30-40 R. du Général Leclerc,

92131 ISSY (FRANCE)

\begin{abstract}
The problem of minimum cost extension of an existing communication network, over a given time period $[0, T]$, is stated and formulated. A two phase solution method is then described, in which: a) an optimal long-range configuration is sought, assuming static routing all over the period $[0, T]$; b) the optimal dynamic routing problem is solved by relaxing the fixed routing assumption, and taking into account the results obtained in phase a).
\end{abstract}

\section{I- INTRODUCTION}

Le problème central en matière de planification de réseaux de Télécommunications peut se résumer de la façon suivante: déterminer une politique optimale d'investissements (au sens du moindre coût actualisé) sur une période donnée $[0, \mathrm{~T}]$, permettant de répondre à une demande en trafic centre à centre croissante au cours du temps.

La connaissance des règles d'acheminement du trafic permet, pour une valeur moyenne souhaitée de la qualité de service, de convertir la demande en trafic en une demande en circuits de transmission (cf. [1], [4]). Cette demande $d_{i j}(t)$, définie pour chaque couple $(i, j)$ de centres (elle peut être nulle pour certains couples) et pour chaque instant $t=0,1, \ldots T$ 
représente le nombre total de circuits de transmission devant être en service à l'instant $t$ entre $i$ et $j$.

Satisfaire la demande $a_{i j}(t)$ suppose:

a) l'installation de matériels de commutation et de modulation en quantité suffisante dans les centres extrémités i et j,

b) l'installation de matêriels de transmission de capacités suffisantes sur un ou plusieurs itinéraires (routages) reliant physiquement $i$ et $j$. (câbles coaxiaux, faisceaux hertziens, guides d'ondes .... avec leurs supports: tranchées, tours hertziennes, etc...)

c) l'installation de materiel de transfert de circuits en chacun des centres intermédiaires de" ces itinêraires (routages).

On voit immédiatement que, pourvu qu'il n'y ait pas création de nouveaux centres, le problème se décompose en deux problèmes indépendants:

$(\underline{P 1})$ : détermination d'une politique optimale d'investissements en matériels de commutation et de modulation dans chaque centre.

$(\underline{P 2})$ : détermination d'une politique optimale d'investissements en matériels de transmission et de transfert sur l'ensemble des artères et des centres.

En effet, les investissements de commutation et de modulation restent. toujours les mêmes, quelle que soit la structure du réseau de transmission. (NB: pour les réseaux téléphoniques, une restriction doit être faite lorsqu'on envisage l'introduction de nouvelles techniques de transmission et de commutation non compatibles avec les anciennes. C'est le cas $p$. ex. de la transmission numérique et de la commutation temporelle).

Ia suite de cet article sera consacrée a la résolution de (P2). Il s'agit essentiellement d'apporter une réponse aux trois questions suivantes:

(Q1): où investir? (détermination de la structure optimale du graphe des extensions).

(Q2) : quoi investir? (parmi tous les matériels de transmission utilisables, quels sont ceux qui, sur chaque artère, permettent de répondre à la demande de la façon la plus économique?).

(Q3): quand investir? (détermination des dates optimales des nouveaux investissements -infrastructures et extensions sur chaque artère du réseau).

Les problèmes de taille réelle (une centaine de centres, plusieurs centaines d'artères) ne pouvant être résolus de façon exacte (il s'agit 
de programmes en nombres entiers comportant des dizaines de milliers de variables entières) la méthode de résolution adoptée se décompose en deux phases:

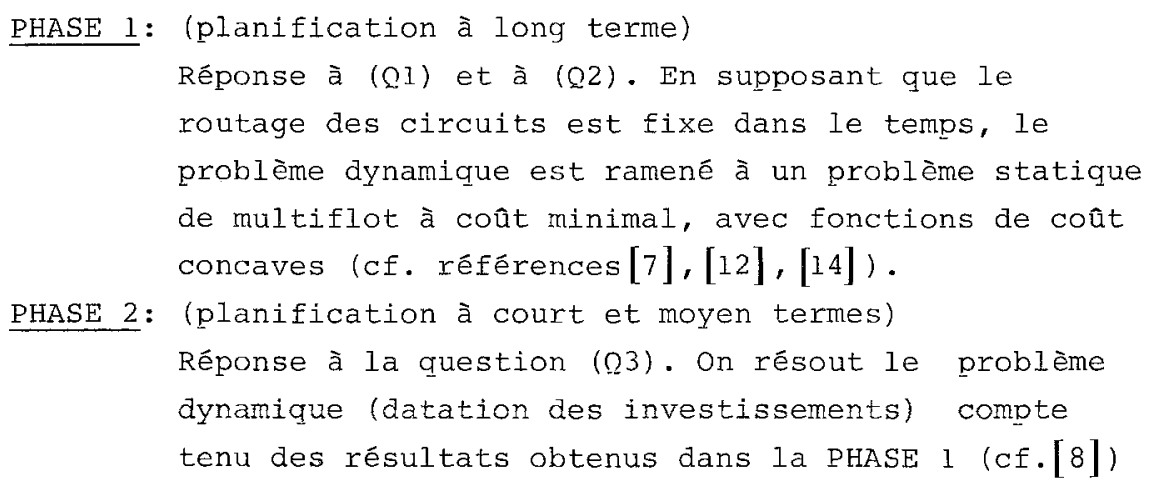

PHASE 2: (planification à court et moyen termes)

Réponse à la question ( $(\Omega 3)$. On résout le problème dynamique (datation des investissements) compte tenu des résultats obtenus dans la PHASE 1 (cf.[8])

Nous commencerons par préciser les données de base indispensables à la résolution du problène (P2).

\section{II- LES DONNEES DE BASE DU PROBLEME}

1) Le réseau étudié est représenté par un graphe non-orienté $\mathrm{G}=[\mathrm{X}, \mathrm{U}]$ où: $\quad \mathrm{X}=1,2, \ldots \mathrm{N}$ est 1 'ensemble des noeuds correspondant aux centres de commutation, de modulation et de transit.

- $U \subset \mathrm{X}^{2}$ est l'ensemble des arêtes $(i, j)$ correspondant aux artères de transmission, soit existantes, soit susceptibles d'être crées.

2) L'intervalle de temps [0,T] (période d'étude) est découpé en T intervalles de durée égale à l'unité (généralement: une année).

3) A chaque instant $t$, le graphe $G$ est parcouru par $K=\frac{N(N-1)}{2}$ flots indépendants, notés $\varphi_{i j}(t)$, pour $i=1, \ldots N \quad j=1, \ldots N$ et $i<j$. Chaque flot $\varphi_{i j}(t)$ a pour extrémités $i$ et j, et sa valeur est une donnée imposée: $d_{i j}(t)$. On supposera en outre que les $d_{i j}(t)$ sont des nombres entiers vërifiant: $d_{i j}(0) \leqslant d_{i j}(1) \leqslant \cdots \leqslant d_{i j}(T)$, $(\forall i=1, \ldots N \quad j=1, \ldots N$ et $i<j)$.

4) Par ailleurs, on donne la liste des différents systèmes de transmission susceptibles d'être installés sur les artères du réseau, avec leurs capacités et leurs coûts. Pour chaque système $s=1,2, \ldots s$, ces coûts dépendent de la longueur 1 de l'artère considérée, et se décomposent en:

- un coût d'infrastructure (élevé) I(s). 
- un coût d'extension $E(s)$ (correspondant à l'équipement d'un canal de transmission supplémentaire)

on note $v(s)$ le nombre d'extensions possibles du système $s$ (nombre total de canaux de transmission) et $Q(s)$ la capacité (en nombre de circuits) d'une extension (d'un canal). La capacité totale du système s est donc: $\nu(s) \cdot Q(s)$.

REMARQUE: pour différents systèmes utilisant la même technique (technique Hertzienne p.ex.) le coût d'infrastructure $I(s)$, qui est le plus important, ne croît que lentement avec la capacité maximale du système $(v(s) . Q(s))$; il en résulte que le coût moyen d'un circuit dêcroit avec la capacité totale installée. Ce phënomène est connu sous le nom d'économie d'échelle (cf. § III ci-dessous). La décroissance du coût moyen suit la loi, dite "des volumes économiques" (cf.[2],[9]):

$$
\frac{I(\mathrm{~s})+\nu(\mathrm{s}) \cdot \mathrm{E}(\mathrm{s})}{\nu(\mathrm{s}) \cdot \Omega(\mathrm{s})}=\mathrm{B}[\nu(\mathrm{s}) \cdot Q(\mathrm{~s})]^{-\mathrm{b}}
$$

où $b$ est un paramètre généralement voisin de 0.5 .

Cette loi, effectivement valable pour la plupart des matériels de transmission connus, ne doit pas être extrapolée hâtivement dans le domaine des fortes capacités, où les systèmes de transmission correspondants ne sont pas bien connus.

5) Les coûts de transfert en un noeud quelconque i $\epsilon \mathrm{X}$, peuvent être considérés comme des fonctions linéaires du nombre de circuits transférés. Soit $f$ le coût de transfert d'un circuit. Pour en tenix compte, il suffit de les intégrer aux coûts d'extension. Ainsi pour un système $s$, on prendra les nouveaux coûts d'extension: $E^{\prime}(s)=E(s)+f . Q(s)$.

6) L'état initial du réseau (à l'année t=0) est connu par la donnée, pour chaque artère $u=(i, j) \in U$, de: la capacité résiduelle disponible (c.a.d. installée mais non encore utilisée) à l'année 0 ; le numéro du dernier système instalıé sur l'artère $u$.

7) On notera $\tau$ le taux d'actualisation.

\section{III- PROGRAMMATION A LONG TERME}

on étuảie l'évolution du réseau sur la période $[0, T]$. Ises routages des flots antérieurs à l'année o (origine de l'étude), ainsi que l'organisation du réseau existant, ne sont pas remis en question. Autrement dit, on s'intéresse simplement au routage des accroissements de demande 
$d_{i j}(t)-d_{i j}(0) \quad(t=1, \ldots T)$. Les capacités résiduelles des systèmes existants, mais non encore saturês à l'année 0 , sont évidemment utilisêes.

\section{1) Les hypothèses}

L'hypothèse fondamentale du modèle à long terme est:

(H1): Les routages sont déterminés une fois pour toutes

à l'année 0 , et invariants dans le temps.

(H1) n'est pas très restrictive en pratique: on constate, en effet, que les routages s'écartent en moyenne assez peu des plus courts chemins géographiques.

Le problème dynamique se ramène ainsi à un problème statique: $\forall i, j(i<j)$, trouver pour tous les flots $\varphi_{i j}(t)$ un routage conduisant à un cô̂t actualisé d'extension minimum.

En plus de l'hypothèse (Hl), nous supposerons une certaine homogèneité dans la croissance des fonctions $d_{i j}(t)$ :

$\underline{(H 2)}$ : Les vitesses de croissance $v_{i j}(t)=d_{i j}(t+1)-d_{i j}(t)$ sont toutes de la forme:

$$
v_{i j}(t)=v_{i j}(0) \cdot g(t)
$$

où $g(t)$ est une fonction (positive) du temps, définie $\forall t=1, \ldots$.

(Autrement dit, les vitesses de croissance des différents flots peuvent être différentes, mais la loi de variation de ces vitesses est supposée la même pour tous les flots).

Pour rechercher, avec les hypothèses (H1) et (H2), un ensemble de routages optimal,il faut avant tout savoir calculer le coût actualisé total de développenent du réseau pour un ensemble de routages donné. Soit un routage défini, pour chaque couple $(i, j)$, par la donnée d'une chaîne $L_{i j}$ reliant $i$ et $j$ dans $G$. Alors, la vitesse de croissance de l'artère $u \in U$ est, à chaque instant $t=1, \ldots T$ :

$y_{u} \cdot g(t)$ où: $\quad \underline{y}_{u}=\sum v_{i j}(0) \quad \&$ somme $\operatorname{sur}$ tous les $\left.i, j t . q \cdot u \epsilon L_{i j}\right)$ est la vitesse de croissance initiale de l'artère u (en nombre de circuits supplémentaires par an).

(N.B.: ce qui précède se généralise immédiatement au cas où plusieurs chaînes $L_{i j}^{1}, L_{i j}^{2}, . .$. sont utilisées pour le routage des circuits entre $i$ et $j$ )

La charge de l'artère u (nombre de circuits supplémentaires par rapport à l'année 0 ) est alors définie à chaque instant par:

$$
Y_{u}(t)=y_{u} \cdot \sum_{j=0}^{t-1} g(j)
$$

Ainsi, l'évolution des besoins futurs sur l'artère u, est caractérisée 
par la donnée d'un seul paramètre $\mathrm{y}_{\mathrm{u}}$.

Le problème se décompose donc, artère par artère: étant donnée la vitesse de croissance initiale $y_{u}$, il s'agit de déterminer une politique d'investissements de coût actualisé minimum sur la période $[0, T]$. on peut alors envisager plusieurs hypothèses sur la fonction $g(t)$.

\section{2) Vitesse de croissance constante (réseau stationnaire).}

Dans ce cas on a: $g(t)=I \quad \forall t$.

Une politique optimale a long terme est alors nécessairement stationnaire, c.a.d. consiste en la répétition indéfinie du même système $s^{*}\left(y_{u}\right)$. Pour trouver $s^{*}\left(y_{u}\right)_{\text {, }}$ il suffit de comparer entre eux les différents systèmes sur une période de même durée.

Pour un système s quelconque, on montre que le côtt actualisé de développement sur $\left[0, t_{0}\right]$ est:

$$
C\left(s, y_{u}\right)=\left[\frac{I(s)}{1-\rho \nu(s)}+\frac{E(s)}{1-\rho}\right]\left[1-\frac{I}{(1+\tau)} t_{0}\right]
$$

avec: $\rho=(i+\tau)^{-\frac{2(s)}{y_{u}}}$

Quand $t_{0} \rightarrow+\infty, C\left(s, \underline{y}_{u}\right)$ tend vers le "cô̂t à l'infini":

$$
C_{\infty}\left(s, y_{u}\right)=\frac{I(s)}{1-\rho \nu(s)}+\frac{E(s)}{1-\rho}
$$

Pour $y_{u}$ donné, le meilleur système est celui qui vérifie:

$$
C^{*}\left(y_{u}\right)=C_{\infty}\left(s^{*}, y_{u}\right)=\underset{s=1, \ldots s}{\operatorname{Min} .}\left\{C_{\infty}\left(s, y_{u}\right)\right\}
$$

et le coût actualisé de développement optimal sur la période $[0, T]$ est:

$$
\Phi_{u}\left(\underline{y}_{u}\right)=C^{*}\left(y_{u}\right) \cdot\left[1-\frac{1}{(1+\tau)^{T}}\right\rceil
$$

Ainsi (à une homothêtie près) la courbe $\Phi_{u}\left(\underline{y}_{u}\right)$ est l'enveloppe inférieure de la famille de courbes $C_{\infty}\left(s, y_{u}\right), s=1, \ldots s$.

Chaque courbe $C_{\infty}\left(s, y_{u}\right)$ a pour ordonnée à l'origine: $I(s)+E(s)$, (qui représente le coût de premier investissement), et admet comme asymptote la droite de pente: $\frac{1}{\tau}\left[\frac{I(s)}{v(s) \cdot Q(s)}+\frac{E(s)}{\Omega(s)}\right]$ passant par
1 'origine.

En fait, dans l'intervalle de validité de chaque système (ensemble des $y$ tels que: $C_{\infty}(s, y)=C^{*}(y)$, chaque courbe peut être assimilée à une droite, et par suite la courbe $\Phi_{u}\left(y_{u}\right)$ peut être considérée comme l'enveloppe inférieure d'une famille finie de droites.

Si la famille des systèmes $s=1, \ldots s$ satisfait la loi "đes volumes économiques" (cf. $\S I I-4$ ), alors on peut montrer que, pour une vitesse 
de croissance initiale $y$, le meilleur système à employer est celui pour lequel la durée de remplissage: $x=\frac{v(s) \cdot Q(s)}{y}$ vérifie:

$$
(1+\tau)^{\mathrm{x}}=1+\frac{\mathrm{x} \cdot \tau}{1-\mathrm{b}} \quad(\mathrm{x} \text { ne dépend donc que de } \tau \text { et de } \mathrm{b}) \text {. }
$$

\section{3) Vitesse de croissance variable.}

Nous envisagerons le cas simple où $g(t)$ est de la forme:

$$
g(t)=(1+\alpha)^{t}
$$

( $\alpha>0$ correspond à un réseau en expansion; $\alpha<0$ correspond à une hypothèse de saturation de la demande).

Notons $\mathcal{y}(\mathrm{y})$ le coût d'installation d'un circuit supplémentaire lorsque la vitesse de croissance de l'artère est $y$. On peut établir et vérifier expérimentalement (cf. [9]) que $y(y)$ suit la loi des "volumes économiques":

$$
\gamma(\mathrm{y})=\mathrm{K}_{1} \cdot \mathrm{y}^{-\mathrm{b}}
$$

Ceci permet de calculer simplement le coût actualisé de đéveloppement de l'artère u sur la période $[0, T]$. En effet:

$$
\Phi_{u}\left(y_{u}\right)=\sum_{t=0}^{T-1} \frac{y \cdot \gamma(y)}{(1+\tau)^{t}}=y_{u} \cdot \gamma\left(y_{u}\right) \cdot \frac{1-\rho^{T}}{1-\rho}
$$

avec: $\rho=\frac{(1+\alpha)^{1-b}}{1+\tau}$, et par suite:

$$
\Phi_{\mathrm{u}}\left(\mathrm{y}_{\mathrm{u}}\right)=\mathrm{K}_{1} \cdot\left(\mathrm{y}_{\mathrm{u}}\right)^{1-\mathrm{b}} \cdot \frac{1-\rho^{\mathrm{T}}}{1-\rho}
$$

Pour les grandes valeurs de $y, f(y)$ ne tend pas vers 0 , mais vers le coût moyen $\mathrm{K}_{2}$ du système de plus grosse capacité. On a alors:

$$
\Phi_{\mathrm{u}}\left(\mathrm{y}_{\mathrm{u}}\right)=\mathrm{K}_{1} \cdot\left(\mathrm{y}_{\mathrm{u}}\right)^{1-\mathrm{b}} \cdot \frac{1-\rho^{\mathrm{T}}}{1-\rho}+\mathrm{K}_{2} \cdot \mathrm{y}_{\mathrm{u}} \cdot \sum_{\mathrm{t}=0}^{\tau-1}\left(\frac{1+\alpha}{1+\tau}\right)^{t}
$$

Dans les deux cas, la fonction $\Phi_{\mathrm{u}}$ est une fonction concave de $\mathrm{y}_{\mathrm{u}}$.

\section{REMARQUE :}

Dans les calculs précédents, il est facile de tenir compte des capacités résiduelles disponibles sur l'artère u à l'année 0 . Supposons que le système existant se sature à l'année $t_{1}>0$, et soit $\mathrm{H}\left(\mathrm{y}_{\mathrm{u}}\right)$ le coût actualisé d'extension jusqu'à $l$ 'année $t_{1}$. En régime de croissance linéaire, par exemple, on aurait:

$$
\Phi_{u}\left(y_{u}\right)=H\left(y_{u}\right)+\frac{1}{(1+\tau)^{t_{1}}} C^{*}\left(y_{u}\right) \cdot\left[1-\frac{1}{(1+\tau)^{T}-t_{1}}\right]
$$




\section{4) Résolution du problème à long terme.}

Quelles que soient les hypothèses de croissance retenues, le problème à long terme se ramène à un problème de multiflot de valeur fixée et de coût minimum. Pour chaque artère $u \in U$ du réseau, le coût actualisé de développement sur la période $[0, T]$ est une fonction $\Phi_{u}\left(y_{u}\right)$ du paramètre $\mathrm{y}_{\mathrm{u}}$ (vitesse de croissance initiale de l'artère).

Dans tous les cas, les fonctions $\Phi_{u}\left(y_{u}\right)$ sont:

- monotones non décroissantes (l'installation de toute capacité supplémentaire est coûteuse);

- concaves (le coût marginal d'un circuit supplémentaire décroît avec la capacité installée).

Avec ces propriétés, il est facile de démontrer qu'un routage optimal est nécessairement un uniroutage (chaque flot $\varphi_{i j} s^{\prime e ́ c o u l e ~ s u r ~ u n ~}$ seul itinéraire). De nombreux travaux ont été consacrés à ce sujet: Dans [12], B. YAGED a proposé une méthode itërative (linéarisations successives) dans laquelle on recherche à chaque étape un routage optimal au sens des coûts marginaux (dérivées des fonctions $\Phi_{u}$ ) relatifs à la solution courante. Dans [14], ZADEH montre que le critère du coût marginal est peu approprié dans le cas où les fonctions $\Phi_{\mathrm{u}}$ présentent une concavité ou un cout fixe importants, et suggère un certain nombre de critères permettant d'améliorer localement une solution.

Dans [6] et [7], on trouvera un algorithme exact (de type "Branch and Bound") pour le cas particulier des fonctions de coût linéaires avec coût fixe (il ne s'applique malheureusement qu'aux réseaux de faibles dimensions: $N \simeq 12$ ).

On y trouvera également un certain nombre de méthodes heuristiques (recherche arborescente "profondeur d'abord") pour le cas général des fonctions de coût non linéaires quelconques; ces méthodes semblent bien adaptées au problème, et conduisent le plus souvent à de très bonnes solutions approchées. Elles ont en outre l'avantage de pouvoir s'appliquer à des problèmes de grandes dimension ( $N=100$ à 200 noeuds).

IV- PROGRAMMATION A COURT ET A MOYEN TERME.

La résolution du problème à long terme a permis de déterminer: - la structure du graphe optimal des extensions $G^{*}=\left[X, U^{*}\right]$ par la liste de ses artères $U^{\star} \in U$. 
- pour chaque artère u $\epsilon \mathrm{U}^{\star}$, une séquence d'investissements $\Sigma_{\mathrm{u}}$ (optimale avec la contrainte du routage fixe dans le temps)

Chaque séquence $\Sigma_{u}$ est définie par:

$P(u)=$ le nombre d'investissements prévus sur $I^{\prime}$ artère $u \in U^{*}$ et, pour $\mathrm{p}=1, \ldots \mathrm{P}(\mathrm{u})$,

$\mathrm{J}(\mathrm{u}, \mathrm{p})=$ le coût total des $\mathrm{p}$ premiers investissements,

$\mathrm{K}(\mathrm{u}, \mathrm{p})=$ la capacité totale installée après le $\mathrm{p}^{\text {ième }}$ investissement.

Dans le modèle a moyen terme, ces résultats sont considérés comme des données de base, c'est à dire que l'on ne remettra en cause sur chaque artère u $\epsilon U^{*}$, ni la nature ni l'ordre des investissements de la séquence $\Sigma_{\mathrm{u}}$, mais uniquement les dates de leur réalisation.

\section{1) Définition d'un êchéancier d'investissements sur $[0, r]$.}

Toute solution du problème (P2) sur la période [0,T] peut être décrite par un échéancier d'investissements $\mathcal{E}$ c'est à dire par la donnée d'un tableau $F(u, t)$ défini $\forall u \in U^{*}$ et $\forall t=0,1, \ldots T-1$. Par définition $F(u, t)$ est le numéro du dernier investissement de la séquence $\Sigma_{u}$ réalisé à l'année $t$. Ainsi, les investissements réalisés à l'année $t$ sont tous ceux dont le numéro est compris entre $F(u, t-1)+1$ et $F(u, t)$. (Par convention $F(u,-1)=0$ ).

Suivant ces notations, le coût actualisé đe l'échéancier $\xi$ est:

$$
\sum_{u \in U^{*}} \sum_{t=0}^{T-1} \frac{J(u, F(u, t))-J(u, F(u, t-1))}{(1+\tau)^{t}}
$$

Le programme à long terme fournit un échéancier $\mathcal{E}^{\circ}=\left\{F^{\circ}(u, t)\right\}$ qui a la particularité d'être optimal lorsque le routage est fixe dans le temps. En abandonnant maintenant l'hypothèse du routage fixe, et en utilisant d'autre part la possibilité de diversifier les routages, certains investissements vont pouvoir être soit retardés, soit supprimés pour conduire à des solutions de coût actualisé inférieur.

\section{2) Réseaux admissibles et échéanciers minimaux.}

Pour $t$ donné, $(0 \leqslant t \leqslant T)$, associons à chaque arète $u \epsilon^{\star}$ de $G^{*}$ la capacité $Y_{u}(t)$ (en nombre de circuits).

on dit que le réseau $R(t)=\left[G^{*}, Y(t)\right]$ est admissible à l'instant $t$ si les capacités $Y_{u}(t) \quad\left(u \in U^{*}\right)$ permettent d'écouler simultanément tous les flots $\varphi_{i j}(t)$. Autrement dit, s'il existe sur $G^{\star}$ un multiflot 
$\left\{\varphi_{i j}(t)\right\}$ compatible avec les capacités $Y_{u}(t)$ (cf. [11]). Evidemment, pour tout échéancier $\mathscr{E}=\{\mathrm{F}(\mathrm{u}, \mathrm{t})\}$ les réseaux $\mathrm{R}(\mathrm{t})=\left[\mathrm{G}^{*}, \mathrm{Y}(\mathrm{t})\right]$ avec: $Y_{u}(t)=K(u, F(u, t-1))$ sont admissibles $(\forall t=1, \ldots T)$. on dit qu'un échéancier $\xi=\{F(u, t)\}$ est minimal à l'instant $t$ si le réseau cesse d'être admissible à l'instant t lorsơu'on supprime un quelconque des investissements qui le composent. On démontre (cf. [5][8]) que tout échéancier de coût actualisé minimum sur la période $[0, T]$ est nécessairement minimal à chaq̣ue instant $t=0,1, \ldots . T$.

\section{3) La méthode.}

Son principe est le suivant: partant de la solution $\varepsilon^{0}$ (déduite du programme à long terme), on engendre une séquence $\xi^{1}, \xi^{2}, \ldots \xi^{n}$ de solutions de coûts actualisés décroissants, jusqu'à obtention d'un échéancier minimal $\varepsilon^{*}$.

Pour cela, on examine successivement toutes les années $t=0,1, \ldots . T-1$, et,à chaque année, on cherche à reporter le maximum d'investissements sur 1 'année suivante.

a) $t=0 ; \mathcal{E}^{\circ}=\left\{\mathrm{F}^{\circ}(\mathrm{u}, \mathrm{t})\right\}$ solution optimale du problème statique.

b) Soit $\xi^{r}=\left\{F^{r}(u, t)\right\}$ la solution courante.

$\forall u \in U^{*}$, déterminer le nombre maximum $q(u)$ d'investissements prévus à l'année $t$ et pouvant être retardés.

Si $q(u)=0 \forall u \in U^{*}$, alors $\mathcal{E}^{r}$ est minimal; aller en e). Sinon:

c) Parmi toutes les possibilités envisagées en b) on détermine la décision la plus économioue - retarder go investissements sur l'artère $u_{0}$ - suivant un critère $\zeta$ (à définir).

d) La nouvelle solution est alors:

$$
\begin{aligned}
& F^{r+}(u, t)=F^{r}(u, t) \quad \forall u \neq u_{0} \\
& F^{r+l}\left(u_{0}, t\right)=F^{r}\left(u_{0}, t\right)-q_{0}
\end{aligned}
$$

Faire: $x \longleftarrow r+1$. Retourner en b).

e) Faire: $t \longleftarrow t+1$, et retourner en b).

Nous avons montrê dans [5] et [8] sur des exemples réels, que cette méthode permet d'améliorer notablement le coût actualisé des solutions obtenues dans le modèle à long terme (avec l'hypothèse des routages invariants dans le temps).

Il existe un grand nombre de possibilités pour le choix du critère $\mathscr{C}$ 
à l'étape c): on pourra par exemple, chercher à différer en priorité 1'investissement le plus coateux; ou encore, l'investissement de cout moyen maximum (rapport entre la valeur de l'investissement et le flot total qu'il permet d'écouler dans la solution courante). Ainsi,si $Y_{u}(t)$ est le nombre de circuits sur l'artère u à l'instant $t$, on cherchera $u_{0}$ et $q_{0}$ maximisant:

$$
\frac{J(u, F(u, t))-J(u, F(u, t)-q)}{Y_{u}(t)-K(u, F(u, t)-q)}
$$

$\forall u \in U^{*}$ et $1 \leqslant q \leqslant q(u)$.

\section{4) Résolution du problème de l'admissibilité.}

La procédure précédente suppose que l'on puisse vérifier rapidement l'admissibilité du réseau dans un grand nombre de configurations différentes.

Le problème de la recherche d'un multiflot compatible sur un graphe $\mathrm{G}^{*}$ non orienté muni de capacitếs est classique et peut être résolu par la programmation linéaire. Cependant, cette technique de résolution présente deux inconvénients: elle est coûteuse en temps de calcul et en encombrement mémoire; elle fournit des solutions fractionnaires.

Dans [8], nous proposons une méthode d'approximations successives (présentant quelques analogies avec la phase I de la méthode "SIMPLEX" en programmation linéaire) qui maintient à chaque itération le caractère entier de la solution, et qui permet de traiter des réseaux de grandes dimensions ( $N=200$ noeuds ou plus). Le temps de calcul est de l'ordre de $\mathrm{N}^{4}$, mais peut être considérablement réduit lorsqu'on dispose d'une bonne solution de départ (Ainsi, la procédure donnée en IV-3 peut être rendue très efficace en utilisant à chaque fois comme solution de départ la dernière solution trouvée). L'expérience montre, par ailleurs, que les résultats fournis s'écartent très peu de l'optimum "en continu" (les différences sont toujours inférieures à $1 \%$ en valeur relative), ce qui permet d'affirmer que les solutions obtenues sont très proches de 1 'optimum en nombres entiers.

REMARQUES :

Lorsque les routages sont variables dans le temps, il en résulte, chaque année, des coûts de gestion supplémentaires (mutation des circuits). Ces coûts peuvent être facilement pris en compte lors de la recherche des routages admissibles (par pënalisation des changements de routage).

La méthode s'étend également sans difficulté au cas où l'on impose des contraintes de sécurité (cf. [8], chap̣. VI). 


\section{$\mathrm{V}-$ CONCLUSION}

Nous avons montré dans [5] que le choix d'une politique d'extension d'un réseau de Télécommunications ne doit pas seulement être guidé par des impératifs à court terme, mais tenir compte également de l'évolution prévisible, à long terme, de la demande et des matériels, afin d'améliorer la rentabilité des investissements effectués. L'approche que nous avons adoptée - planification à long terme, puis utilisation des rêsultats obtenus pour la planification à moyen terme se trouve ainsi justifiée.

Terminons en signalant que les mêthodes exposées dans cet article commencent à rentrer en exploitation pour la préparation des plans d'investissements en transmission 1976 et 1977 sur le réseau interurbain français.

\section{REFERENCES:}

[1] AYMAR (J.P.) "VOICI: un programme permettant d'évaluer les besoins futurs dans un réseau interurbain". Echo des Recherches (C.N.E.T.) Octobre 1970.

[2] ELLIS (L.W.) "La loi des volumes économiques appliquée aux Télécommunications". Rev. Telecom. $N^{\circ} 50,1$ (1975).

[3] HARTMAN (J.K.) LASDON (L.S.) "A generalized Upper Bounding algorithm for multiconmodity network flow problems". Networks, 1 (1972).

[4] MAURY (J.P.) "Planification des réseaux de Télécommunications". Annales des Télécom. 25, N5-6 (1970).

[5] MINOUX (M.) "Multiflots dynamiques de coût actualisé minimal". Annales des Télécom. 30, $\mathrm{N}^{\circ} 1-2$ (1975).

[6] MINOUX (M.) "Recherche de la configuration optimale d'un réseau de Télécommunications". (à paraltre).

[7] MINOUX (M.) "Recherche de la configuration optimale d'un réseau de Télécommunications avec fonctions de coût concaves". Annales des Télécorn. 29, $\mathrm{N}^{\circ} 1-2$ (1974).

[8] MINOUX (M.) "Planification à court et moyen terme d'un réseau de Télécomunications". Annales des Télécom. 29, No11-12 (1974).

[9] MOULON (M.) "Comparaison économique des systèmes à large bande: la méthode du coût équivalent". Revue F.I.T.C.E. Mai-Juin 1972.

[10] SCOTT (A.J.) "The optimal network problem: some computationnal procedures". Transp. Res. 3,(1969) pp. 201-210.

[1] TOMLIN (J.A.) "Minimum cost multicommodity network flows". Ops. Res. 14, $\mathrm{N}^{\circ} \mathrm{I}(1966)$.

[12] YAGED (B.) "Minimum cost routing for static network models". Networks 1, $\mathrm{N}^{\circ} 2$ (1971).

[13] YAGED (B.) "Minimum cost routing for dynamic network models". Networks 3, $\mathrm{N}^{\circ} 3$ (1973).

[14] ZADEH (N.)" "On building minimum cost communication networks". Networks 3, $\mathrm{N}^{\circ} 4$ (1973).

[15] ZADEH (N.) "On building minimum cost communication networks over time". Networks $4, \mathrm{~N}^{\circ} 1(1974)$. 